\title{
Suelos de la finca SNAKY-URACCAN Costa Caribe Norte de Nicaragua
}

Fidel Wilson Williams ${ }^{1}$ Joshua Sujo Herrera ${ }^{2}$ Enrique Cordón Suárez ${ }^{3}$

\section{Resumen}

Se estudió el suelo en la finca académica Snaky-URACCAN, en la comunidad de MossPam, municipio de Waspam, con el fin de valorar las propiedades físicas y químicas del suelo en los diferentes usos que tiene la finca.

La metodología fue mediante la recolección de muestras por el sistema zigzag. Se recolectó en cada uno de los vértices, realizando sub muestras a una distancia 10 metros con una profundidad de $20 \mathrm{~cm}$ superficial y 40 sub-superficial. En el área de bosque se recolectaron 45 sub-muestras, en el área agrícola y pecuaria se recolectaron 60 sub-muestras por los dos usos. Posteriormente se realizaron 7 muestras compuestas en cada uso para su análisis en el laboratorio LAQUISA S.A, ubicada en departamento de León.

Los resultados muestran que los suelos de la finca Snaky-URACCAN han presentado características físicas apropiadas para el desarrollo de cualquier cultivo. Su densidad aparente es baja, con predominio de textura franco arenoso, los colores predominantes de suelo son: amarillo rojizos a pardo. Sin embargo, sus propiedades químicas presentan deficiencias significativas. Los macronutrientes como el nitrógeno y el fósforo presentaron niveles muy bajos, no así el potasio que presentó altos niveles. En el caso de los micronutrientes, el calcio presentó niveles regulares, mientras que el hierro, magnesio y manganeso presentaron niveles altos que coinciden con la alta acidez del suelo.

Palabras clave: Textura; densidad aparente, $\mathrm{pH}$; materia orgánica; macro y micro nutrientes.

\section{Summary}

The soil in the academic farm Snaky-URACCAN was studied, in the community of MossPam, Waspam municipality, in order to assess the physical and chemical properties of the soil in the different uses of the farm.

1 Ingeniero Agroforestal. Correo: fidirams.9o@gmail.com

2 Ingeniero Agroforestal. Correo: sujojoshua@yahoo.es

3 Doctor en Agroforestería Tropical, Vicerrector de la Universidad de las Regiones Autónomas de la Costa Caribe Nicaragüense Recinto Universitario de Bilwi. Correo: enrique.cordon@uraccan.edu.ni

Recibido: 07/03/2017 Aprobado: 23/11/2017

Wilson-Williams, F., Sujo-Herrera, J., \& Cordón-Suárez, E. (2017). Suelos de la finca SNAKY-URACCAN Costa Caribe Norte de Nicaragua. 
The methodology used was through the collection of samples by the zigzag system. It was collected in each one of the vertices, making sub samples at a distance of 10 meters with a depth of $20 \mathrm{~cm}$ superficial and 40 sub-superficial. In the forest area, 45 sub-samples were collected, in the agricultural and livestock area, 6o sub-samples were collected for the two uses. Subsequently, 7 combined samples were made in each use for analysis in the LAQUISA S.A. Laboratory located in the department of León.

The results show that the soils of the Snaky-URACCAN Farm have presented physical characteristics appropriate for the development of any crop. Its apparent density is low, with a predominance of sandy loam texture, the predominant soil colors are: reddish brown to brown. However, its chemical properties present significant deficiencies. Macronutrients such as nitrogen and phosphorus showed very low levels, but potassium did show high levels. In the case of micronutrients, calcium presented regular levels, while iron, magnesium and manganese showed high levels that coincide with the high acidity of the soil.

Key Words: texture; bulk density, $\mathrm{pH}$; organic material; macro and micro nutrients.

\section{Introducción}

Esta investigación es resultado de un estudio de suelo realizado en la finca académica Snaky URACCAN, en la comunidad de Moss Pam, municipio de Waspam, con el fin de valorar las propiedades físicas y químicas (textura, color y densidad aparente, $\mathrm{pH}$, materia orgánica, macro y micro nutrientes), del suelo en los diferentes usos que tiene la finca.

La finca académica SNAKI-URACCAN se encuentra ubicada en la comunidad de Moospam, municipio de Waspam río Coco y cuenta con un área de 107 hectáreas. Su uso está distribuido en tres modelos productivos, siendo pecuaria, forestal y agrícola, sin embargo esta distribución se hizo sin considerar las características físicas y químicas del suelo. El objetivo de la finca es experimental y de utilización para el desarrollo de prácticas de campo de los estudiantes y docentes la universidad URACCAN, principalmente los de la carrera de ingeniería agroforestal.

La importancia de realizar esta investigación es la de conocer el grado de fertilidad de la finca, considerando que no cuenta con estudios detallados de suelo y que permita conocer la calidad de los nutrientes que hay en suelo. Los resultados obtenidos de esta investigación le darán un mejor uso de suelo para sus diferentes actividades. 


\section{Revisión de literatura}

\section{Definición de suelo}

Según Buol, S. W.; Hole, F. D. and McCracken, R. J. (2007), el suelo a la parte superficial de la tierra, biológicamente activa, que tiende a desarrollarse en la superficie de las emergidas por la influencia de la intemperie y de los seres vivos. En este sentido se puede entender que los suelos son sistemas complejos donde ocurre una diversidad de procesos químicos, físicos y biológicos y que derivan una gran diversidad de suelos existentes en la tierra.

\section{Componentes del suelo}

Según De la Rosa (2006), los suelos contienen cuatro elementos básicos, siendo los siguientes:

Elementos minerales: Representan normalmente la mitad del volumen del suelo, aunque aparenten constituir la totalidad del mismo; estos elementos minerales se encuentran en diferentes tamaños y suelen ser clasificados, de mayor a menor, como arena, limo o arcilla. La composición mineral del suelo determina sus propiedades físicas, condiciona y es condicionado por las formas de vida presentes.

El agua: Ésta representa normalmente un cuarto del volumen del suelo, aunque la cantidad exacta puede variar grandemente en función de la estación del año y del tipo de suelo. Con muy poca agua el suelo está desecado, y con mucha agua saturado.

El aire: Se dice que en suelos bien agregados puede representar otro cuarto del volumen, conteniendo oxígeno, hidrógeno, nitrógeno y carbono en forma gaseosa. Cuanto mayor sea el espacio poroso del suelo más grande será su capacidad para retener agua y aire que beneficie a las plantas, así como a cualquier otra flora o fauna presente en el mismo.

El material orgánico: Respecto a lo expuesto por De La Rosa se entiende que éste, regularmente, representa sólo una pequeña porción del suelo, entre un porcentaje de 1 a 6, aunque puede ser mayor en algunos casos. Este cuarto componente está formado por materia orgánica no viva, derivada del desarrollo, reproducción, muerte y descomposición de plantas, animales y microbios, existiendo en el suelo como humus o como otro material inanimado. Una inmensa variedad de fauna y flora viva a la que se conoce como biota del suelo. Las raíces de las plantas soportadas por el suelo. 


\section{Propiedades físicas}

Según Tarbuck y Lutgens (1999), las propiedades físicas de los suelos son aquellas relacionadas con la organización estructural de un suelo, que son utilizadas en su descripción o determinadas en el laboratorio, y que equivale a su arquitectura o composición textural.

\section{Textura del suelo}

Según Warren, F (1985), la textura del suelo es una propiedad física permanente que se refiere a la expresión porcentual de las fracciones granulométricas arena, limo y arcilla, a la combinación de estas tres se determina una clase textural. De igual manera Marconi (2008), explica que la textura es la cantidad (\%) y calidad de las partículas minerales que contiene el suelo.

Suelos arenosos: son aquellos que son ligeros y filtran el agua rápidamente. Además, tienen poca materia orgánica, por lo que no son muy fértiles.

Suelos arcillosos: estos son suelos pesados que casi no filtran el agua, son pegajosos, plástico en estado húmedo y poseen muchos nutrientes y materia orgánica. $\mathrm{Al}$ respecto Simmons, Tárrano y Pinto (2002), indican que la arcilla está formada de partículas minerales pequeñas del suelo, menores de $0.002 \mathrm{~mm}$ de diámetro. Por lo tanto, el suelo arcilloso debería de haber porcentajes de 40 o más de arcilla, menos de 45 de arena y menos de 40 en suelo limoso.

Suelos limosos: Es aquel que en la mayoría de los casos es estéril, pedregoso y filtra el agua con rapidez; además, la materia orgánica que contiene, se descompone con gran celeridad. Los autores antes mencionados reflejan que un suelo limoso es aquel con partículas del suelo, de tamaño intermedio entre las de arena y arcilla y que poseen de entre 0.05 a $0.002 \mathrm{~mm}$ de diámetro. Por lo tanto, el suelo limoso debería de contener un porcentaje de 80 o más de limo y menos del 12 de Arcilla.

\section{Color del suelo}

El color del suelo es la característica más fácil de detectar por medio de la observación. Tradicionalmente a los suelos se les denominan según el color que presentan como: suelos rojos, suelos pardos, suelos podzoles o suelos cenicientos, tierra rosa, etc.

Es importante aclarar que un suelo no es mejor o peor por presentar un color determinado, el color no tiene valor intrínseco, pero es muy importante en relación con procesos edáficos y propiedades del suelo. 


\section{Densidad aparente del suelo}

Se refiere al peso seco en gramos de materiales sólidos dentro de un volumen definido. Como el suelo está constituido por partículas que difieren en tamaño y forma, lo cual incluye los espacios porosos entre las partículas, se establece las relaciones de masa por volumen y originan dos conceptos, siendo la densidad aparente y densidad real o de partículas (Keller, T.; Håkansson, I. 2010).

Tiene un ámbito normal de valores que es influenciado por el tipo de material parental que dio origen al suelo. Se define como densidad aparente, a la relación: peso de suelo seco al horno (pss) a $150{ }^{\circ} \mathrm{C}$ por 24 horas/volumen total de la muestra del suelo $(\mathrm{Vc})$ incluyendo el espacio poroso.

Tabla 1: Valores de densidad aparente del suelo.

\begin{tabular}{|c|c|c|c|c|c|}
\hline $\begin{array}{c}\text { Unidad de la (Da) } \mathbf{g} / \\
\mathbf{c m} 3\end{array}$ & $<\mathbf{1 . 0}$ & $\mathbf{1 . 0 - 1 . 2}$ & $\mathbf{1 . 2 - 1 . 4 5}$ & $\mathbf{1 . 4 5 - 1 . 6 0}$ & $>\mathbf{1 . 6 0}$ \\
\hline Clasificación & $\begin{array}{c}\text { Muy } \\
\text { Bajo }\end{array}$ & Bajo & Medio & Alto & $\begin{array}{c}\text { Muy } \\
\text { alto }\end{array}$ \\
\hline
\end{tabular}

Taboada \& Álvarez (2008).

Tabla 2: Valores de densidad aparente según la textura.

\begin{tabular}{|c|c|c|c|c|}
\hline Textura & Arenas & Francos & Arcillas & $\begin{array}{c}\text { Suelos } \\
\text { orgánicos }\end{array}$ \\
\hline $\begin{array}{c}\text { Densidad apa- } \\
\text { rente }(\mathrm{g} / \mathrm{cm} 3)\end{array}$ & 1.6 a 1.7 & 1.3 a 1.4 & 1.0 a 1.2 & 0.7 a 1.0 \\
\hline \multicolumn{4}{|c|}{ Dexter et al., (2004). }
\end{tabular}

Densidad Real: También llamada Densidad de Partículas o Densidad Bruta, "se entiende por esta a la relación entre el peso de las partículas y el volumen que ocupan, sin incluir el espacio poroso". En otras palabras, es la cantidad de masa de sólidos que existe por unidad de volumen.

\section{Propiedades químicas del suelo}

Según Foth (1985), los elementos químicos del suelo pueden estar contenidos en la fase solida y liquida formando parte de la estructura de los minerales o en los compuestos orgánicos.

\section{pH en el suelo}

Según Fasbender (1985), la reacción del suelo se refiere al agrado de acidez o alcalinidad (basicidad) del suelo y se expresan en $\mathrm{pH}$; el cual tiene una gran influencia en muchas de sus propiedades físicas, químicas y biológicas. 
De acuerdo a Fassbender (1982), los suelos con pH entre 5,5 y 6,5; los suelos con pH entre 6,1 y 6,5 presentan las condiciones casi óptimas para la nutrición de la mayoría de las plantas. En los suelos con $\mathrm{pH}$ entre 5,5 y 6 las principales limitaciones para su uso agropecuario se relacionan con problemas de nutrición ocasionados por desbalances entre nutrientes o por diferencias de ellos en el suelo.

\section{Materia orgánica}

Según Gutiérrez (2010), son los residuos de plantas y animales descompuestos. La materia orgánica da al suelo algunos alimentos que las plantas necesitan para su crecimiento y producción. La materia orgánica mejora las condiciones del suelo para el buen desarrollo de los cultivos. Los rangos de materia orgánica es el siguiente: menor de 1.8 es bajo, de 1.9-4.2 es medio y más de 4.2 es alto.

\section{Macro nutriente}

Es un grupo de minerales en las cuales las plantas los requieren en cantidades considerables. Entre ellos tenemos el nitrógeno, fósforo, potasio y azufre.

\section{Nitrógeno}

Es un nutrientes muy útil para el crecimiento y desarrollo del las plantas. Favorece el crecimiento vegetativo, produce suculencia, da el color verde a las hojas, gobierna en las plantas el uso de potasio, fósforo y otros. Un exceso de este elemento retarda la maduración, debilita la salud de las plantas.

Tabla 3: Rangos de Nitrógeno en el suelo.

\begin{tabular}{|c|c|c|c|c|c|}
\hline $\begin{array}{c}\text { Mayor de } \\
0.18 \%\end{array}$ & 0.15 a $0.18 \%$ & $\begin{array}{l}0.10 \mathrm{a} \\
0.15 \%\end{array}$ & $\begin{array}{l}0.08 \mathrm{a} \\
0.10 \%\end{array}$ & $\begin{array}{l}0.05 a \\
0.08 \%\end{array}$ & $\begin{array}{c}\text { Menor de } \\
0.05 \%\end{array}$ \\
\hline Alto & L. Alto & Normal & L. Bajo & Bajo & $\begin{array}{l}\text { Muy } \\
\text { bajo }\end{array}$ \\
\hline
\end{tabular}

López R., J. Y López M., J. 1990.

\section{Fósforo}

Es el segundo macro nutriente de mayor importancia, ya que interviene en numerosos procesos bioquímicos a nivel celular, contribuye a las raíces y a las plántulas a desarrollarse rápidamente y mejora su resistencia a las bajas temperaturas. También incrementa la eficiencia del uso del agua y contribuye a la resistencia de algunas plantas a enfermedades. 
Los rangos establecidos (Etchevers B., J. P. Anzastiga, V. Volke y G. Etchevers. 1986.) es el siguiente: menos de $10 \mathrm{ppm}$, es bajo y como tal perjudica los rendimientos de los cultivos, de 11-20 ppm, es medio y mayor de $21 \mathrm{ppm}$, es alto.

\section{Potasio}

También es considerado un elemento primario y esencial para los cultivos. Su rol es importante en la activación enzimática, fotosíntesis, síntesis de proteínas y carbohidratos, balance de agua, en el crecimiento meristemáticos, favorece el crecimiento vegetativo, fructificación, maduración y calidad de los frutos (Etchevers B. J. 1991).

Tabla 4: Niveles de Potasio en el suelo.

\begin{tabular}{|l|l|}
\hline \multicolumn{1}{|c|}{ Rango (meq/100g suelo } & \multicolumn{1}{c|}{ Clasificación } \\
\hline$<0.2$ & Pobre \\
\hline $0.2-0.3$ & Medio \\
\hline$>0.3 \quad$ & Alto \\
\hline
\end{tabular}

\section{Micronutrientes}

\section{Magnesio}

Se encuentra en la solución del suelo y se absorbe en las superficies de las arcillas y la materia orgánica. Los suelos generalmente contienen menos $\mathrm{Mg}$ que $\mathrm{Ca}$ debido a que el $\mathrm{Mg}$ no es absorbido tan fuertemente como el Ca por los coloides del suelo y puede perderse más fácilmente por lixiviación (Sadzawka y Campillo, 1999). Cuando en los suelos se encuentran rangos menores $2 \mathrm{meq} / 100$ gr de suelo, este es considerado bajo para el buen rendimiento de los cultivos, 2.1-10 meq/10o gr de suelo es medio y mayor de $10 \mathrm{meq} / 100$ gr de suelo, es alto.

\section{Manganeso}

La carencia de Manganeso ofrece síntomas parecidos a los del hierro: hojas amarillas entre los nervios que permanecen verdes. Se puede diferenciar porque en este caso aparece una aureola verde alrededor de los nervios. Las causas de la carencia es por suelos calcáreos y por suelos arenosos muy lavados (Suárez, 1991).

Según Charlton; Hampton y Scoth (1986), el magnesio en el suelo puede ser menos de $5 \mathrm{ppm}$ y es considerado bajo y malo para la agricultura, medio de 6-50-12 ppm, y alto mayor a $20 \mathrm{ppm}$. 


\section{Hierro}

El hierro se encuentra en la naturaleza en cantidad suficiente formando distintos compuestos como óxidos e hidróxidos. Sin embargo, la cantidad total no se correlaciona con la cantidad disponible para las plantas (Cisternas, 1987)

La coloración de los suelos es debida a su presencia son los colores de suelos amarillo-pardo, rojos y rojizos. Es común ver en el sector de la ciudad de Bilwi el predominio de estos colores.

El hierro en los suelos puede ser de $10 \mathrm{ppm}$ el cual es considerado bajo y malo para la agricultura, de 11-100 ppm es considerado medio y de 100-130 ppm es considerado alto y mayor de $130 \mathrm{ppm}$ es considerado muy alto (Cuevas y Balocchi,1983).

\section{Zinc}

La deficiencia en $\mathrm{Zn}$ se da en una amplia variedad de suelos como son los sueltos, los calcáreos, margosos y arenosos pobres en materia orgánica, aunque sobre todo en estos últimos. En cuanto al $\mathrm{pH}$, el $\mathrm{Zn}$ se encuentra más disponible en los suelos ácidos que en los alcalinos, siendo su mínima disponibilidad para $\mathrm{pH}$ por encima de 7 (Ortega, 1992.).

En el suelo se encuentra en rangos siguientes, menor a 3 ppm, es considerado bajo y afecta el rendimiento de los cultivos, rangos de $3.1-10$ ppm es considerado medio y mayor de 10 ppm es considerado alto (Paladines y Muñoz, 1982.

\section{Cobre}

El cobre es un elemento secundario que ayuda a la coloración en las hojas y al desarrollo radicular. Se presenta la carencia en suelos calcáreos básicamente. En el suelo se encuentra en rangos de $2 \mathrm{ppm}$, es considerado bajo y afecta el rendimiento de los cultivos, rangos de 3-20 ppm es considerado medio y mayor de $120 \mathrm{ppm}$, es considerado alto (Ruz y Campillo, 1996).

\section{Materiales y métodos}

\section{Tipo de Estudio}

La investigación es de tipo cuantitativo considerando que nos permite conocer la calidad y cantidad de los elementos minerales presentes en el suelo. 


\section{Universo y muestra}

El universo lo constituyen las 107 hectáreas de extensión de la finca académica "SNAKY" distribuidas en tres usos actuales que son: uso bosque, agrícola y pecuario. De manera general se levantaron un total de 105 muestras de suelo utilizando en método del zigzag (ver figura 1). En el área de bosque se levantaron 45 muestras equidistantes $10 \mathrm{~m}$ por muestra. En el área agrícola se levantaron 30 muestras a una distancia de 5 metros por muestra y finalmente en el área pecuaria se levantaron 30 muestras a una distancia de 5 metros entre cada una.

Para el envío al laboratorio y hacer el análisis químico de las muestras, se hizo una mezcla compuesta de las muestras recogidas para cada sector por separado (forestal, agrícola y pecuario). Posteriormente, se obtuvieron tres muestras únicas para el sector forestal, dos muestras para el sector agrícola y dos para el sector pecuario. En total se conformaron 7 muestras compuestas para enviarlas al laboratorio de un total de 105 levantadas en el campo.

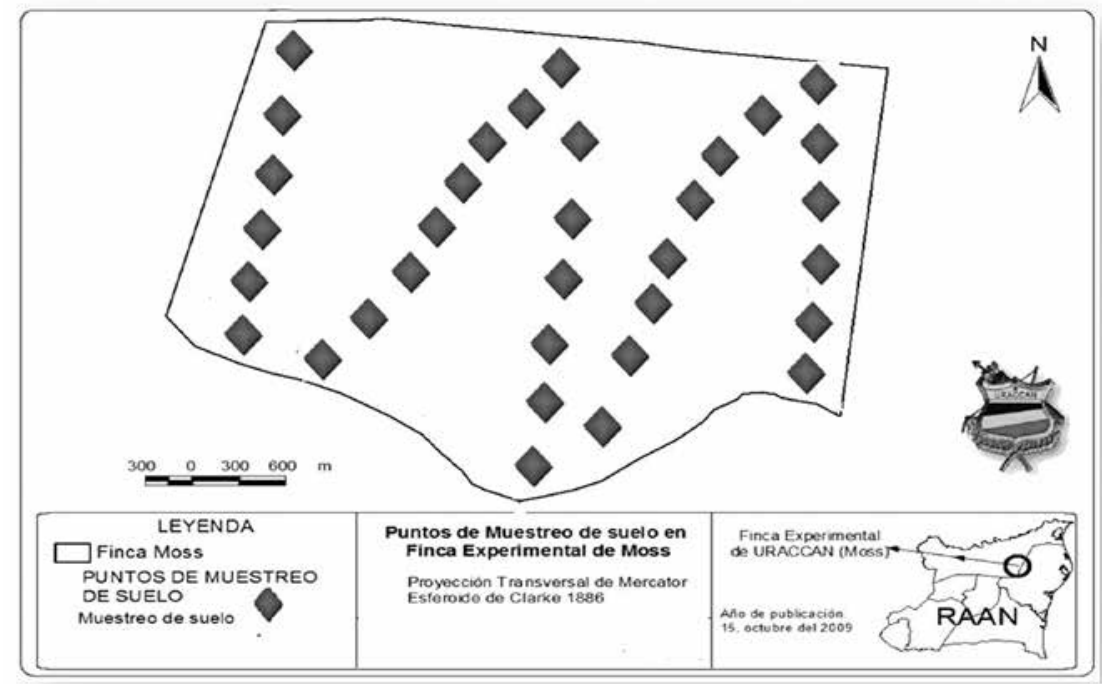

Figura 1: Levantamiento de muestras en el suelo

\section{Densidad aparente}

Para determinar la densidad aparente se hizo lo siguiente: Se introdujo un cilindro al suelo con la ayuda de un pedazo madera, posteriormente se retiró el cilindro con la pala y se limpió el sobrante de suelo que quedó en los bordes con ayuda de un cuchillo, luego se introdujo en un balde plástico, una vez realizada en cada muestra se hizo una remoción en el suelo para posteriormente guardarlas y etiquetarlas en una bolsa plástica para su debido análisis. 
De acuerdo a Sánchez (2005), la densidad aparente en determinado estado de compactación permite transformar peso a volumen o viceversa. Esto permite conocer el grado de compactación o huecos que posee el suelo.

\section{Textura}

De acuerdo al autor antes mencionado, la textura indica el contenido relativo de partículas de diferente tamaño, como la arena, el limo y la arcilla en el suelo. La textura tiene que ver con la facilidad con que se puede trabajar el suelo, la cantidad de agua y aire que retiene y la velocidad con que el agua penetra en el suelo y lo atraviesa. La textura se realizó mediante una perforación al suelo con la ayuda del barreno tanto superficial como sub superficial.

\section{Determinación del color del suelo}

Para determinar el color del suelo se tomó una porción de suelo de cada sub muestras realizadas tanto superficiales y sub superficial para luego compararlas con la tabla munsell.

Según Ovalles (2006), el color del suelo es una de las características morfológicas más importantes del suelo, es la más obvia y fácil de determinar. Permite identificar distintas clases de suelos. El objetivo de este trabajo es destacar el significado del color del suelo, así como las relaciones que él tiene con condiciones edáficas particulares. Se describen los sistemas más empleados en la designación del color, siendo el Sistema Munsell el de mayor uso.

\section{pH}

De acuerdo a Velarde (2003), el pH es una escala numérica que mide el grado de acidez o alcalinidad de una substancia. Esta escala se mueve desde o a 14, una sustancia menos de 7 se dice que es ácida, por encima de 7 se dice que es alcalina.

$\mathrm{El} \mathrm{pH}$ fue estimado con cinta peachimétrica. Inicialmente se tomaron muestras de suelo superficial y sub-superficial, en cada muestra levantada, las cuales fueron desmenuzadas y disueltas con agua purificada en un recipiente, para luego introducir la cinta y leer su $\mathrm{pH}$.

\section{Materia orgánica}

Para la realización de la materia orgánica se hizo por medio del método Walkley y Black, se estima el contenido de carbono orgánico total de una muestra de suelo, completo o de alguna de sus fracciones. Es el método más utilizado en los laboratorios edafológicos para evaluar la materia orgánica del suelo. 


\section{Determinación de macro y micro nutrientes}

De manera general la muestra de suelo es digerida en presencia de ácido sulfúrico concentrado, sulfato de potasio y sulfato cúprico hasta desprendimiento de humos blancos y que la solución sea transparente e incolora o de un tono amarillo paja. El residuo es enfriado, diluido y llevado a condiciones alcalinas para la determinación del amonio. El amonio destilado se cuantifica volumétricamente. Este procedimiento fue similar en con los demás elementos en estudio.

\section{Resultados y discusión}

\section{Textura}

\section{El estudio de la textura en los suelos de la finca Snaky se refleja en la tabla.}

Tabla 5: Textura por ecosistema finca Snaky.

\begin{tabular}{|l|l|l|l|l|}
\hline \multirow{2}{*}{ Muestras de ecosistemas } & \multicolumn{3}{c|}{ Textura } & \multirow{2}{*}{ Valoración } \\
\cline { 2 - 4 } & Arcilla \% & Limo \% & Arena $\%$ & \\
\hline Sector Bosque 1 & 16.64 & 30.92 & 52.44 & Franco arenoso \\
\hline Sector Bosque 2 & 8.28 & 25.28 & 66.44 & Franco arenoso \\
\hline Sector Bosque 3 & 4.28 & 15.28 & 80.44 & Franco arenoso \\
\hline $\begin{array}{l}\text { Sector agrícola 1 } \\
\text { (Cítrico, plátano, cacao) }\end{array}$ & 16.64 & 32.92 & 50.44 & Franco arenoso \\
\hline $\begin{array}{l}\text { Sector agrícola 2 } \\
\text { (Cítricos plátano cacao) }\end{array}$ & 12.64 & 22.92 & 64.44 & Franco arenoso \\
\hline Sector pecuario 1 & 22.28 & 28.28 & 49.44 & Franco arenoso \\
\hline Sector pecuario 2 & 12.28 & 19.28 & 68.44 & Franco arenoso \\
\hline
\end{tabular}

Cabe mencionar que los suelos francos y franco arenoso son característicos de las vegas de los ríos considerando que la finca académica Snaky se encuentra en la vega el río Wawa. Estos suelos generalmente tienen mucho drenaje y tienden a tener una buena aireación, también ayuda a los organismos en el suelo a sobrevivir y frecuentemente benefician a las plantas ayudándolas a absorber los nutrientes. Una de las desventajas de estos es que se podrían lavar más fácilmente por su textura arenosa predominante, lo cual significa que terminan siendo pobres con el tiempo.

\section{Densidad aparente}

En el caso de los suelos de la finca Snaky, los resultados de la prueba de laboratorio reflejaron lo siguiente en la tabla No. 6: 
Tabla 6: Densidad aparente por ecosistemas finca Snaky

\begin{tabular}{|c|c|c|c|}
\hline Muestras de ecosistemas & $\begin{array}{l}\text { Densidad } \\
\text { Aparente (g/ } \\
\mathrm{ml})\end{array}$ & Valoración & Características del sitio \\
\hline Sector Bosque 1 & 1.17 & \multirow{4}{*}{ Bajo } & \multirow{4}{*}{$\begin{array}{l}\text { Bosques secundarios } \\
\text { y afectados por hura- } \\
\text { cán en el } 2007\end{array}$} \\
\hline Sector Bosque 2 & 1.16 & & \\
\hline Sector Bosque 3 & 1.11 & & \\
\hline Promedio & 1.14 & & \\
\hline $\begin{array}{l}\text { Sector agrícola } 1 \\
\text { (Cítrico, plátano, cacao) }\end{array}$ & 1.15 & \multirow{3}{*}{ Bajo } & \multirow{3}{*}{$\begin{array}{l}\text { Cultivos de cítricos, } \\
\text { plátanos y cacao. }\end{array}$} \\
\hline $\begin{array}{l}\text { Sector agrícola } 2 \\
\text { (Cítricos plátano cacao) }\end{array}$ & 1.13 & & \\
\hline Promedio & 1.14 & & \\
\hline Sector pecuario 1 & 1.12 & \multirow{3}{*}{ Bajo } & \multirow{3}{*}{$\begin{array}{l}\text { Ganadería mayor, con } \\
\text { pastos mejorados } \\
\text { (Taiwán, Bracharia y } \\
\text { Brizanta). }\end{array}$} \\
\hline Sector pecuario 2 & 1.13 & & \\
\hline Promedio & 1.12 & & \\
\hline Promedio general & 1.13 & Bajo & \\
\hline
\end{tabular}

Generalmente se maneja que los suelos de bosques tienen una densidad aparente más baja que los suelos de áreas agrícolas o pecuarias; no obstante, en los suelos de la finca Snaky, la densidad aparente es relativamente similar en los tres ecosistemas estudiados y también coincidentes con el tipo de suelo franco arenoso.

Es muy probable de que debido a que los objetivos de la finca son académicos y no de explotación intensiva, o sea que no se utiliza ningún tipo de maquinaria, por tanto aún no sufren daños los suelos y mantienen buena estabilidad los agregados, buena humedad y buena capacidad de infiltración.

\section{Color de los suelos}

Tabla 7: Colores de los suelos por ecosistemas.

\begin{tabular}{|l|l|l|l|}
\hline Muestras de suelo por ecosistemas & $\begin{array}{c}\text { Color suelo superficial } \\
(\mathbf{0}-\mathbf{1 0} \mathbf{c m})\end{array}$ & $\begin{array}{c}\text { Color suelo sub superficial } \\
(\mathbf{1 0 - 2 0} \mathbf{c m})\end{array}$ & Valoración Superficial y sub superficial \\
\hline Sector Bosque 1 & $7.5 \mathrm{YR}$ & $5 \mathrm{YR}$ & $\begin{array}{l}\text { Superficial pardo. } \\
\text { Sub superficial amarillo rojizo. }\end{array}$ \\
\hline Sector Bosque 2 & $10 \mathrm{YR}$ & $7,5 \mathrm{R}$ & $\begin{array}{l}\text { Superficial amarillo. } \\
\text { Sub superficial pardo. }\end{array}$ \\
\hline
\end{tabular}




\begin{tabular}{|c|l|l|l|}
\hline Muestras de suelo por ecosistemas & $\begin{array}{c}\text { Color suelo superficial } \\
(\mathbf{0}-\mathbf{1 0} \mathbf{c m})\end{array}$ & $\begin{array}{c}\text { Color suelo sub superficial } \\
(\mathbf{1 0 - 2 0 \mathbf { c m } )}\end{array}$ & Valoración Superficial y sub superficial \\
\hline Sector Bosque 3 & $2.5 \mathrm{YR}$ & $7,5 \mathrm{YR}$ & $\begin{array}{c}\text { Superficial Rojo. Y oscuro } \\
\text { sub. Superficial pardo. }\end{array}$ \\
\hline Muestras de suelo por ecosistemas & $\begin{array}{c}\text { Color suelo superficial } \\
(\mathbf{0}-\mathbf{1 0} \mathbf{c m})\end{array}$ & $\begin{array}{c}\text { Color suelo sub superficial } \\
(\mathbf{1 0 - 2 0} \mathbf{c m})\end{array}$ & Valoración Superficial y sub superficial \\
\hline Sector agrícola 1 & $7.5 \mathrm{YR}$ & $5 \mathrm{YR}$ & $\begin{array}{c}\text { Superficial pardo. } \\
\text { Sub superficial amarillo rojizo. }\end{array}$ \\
\hline Sector agrícola 2 & $2.5 \mathrm{YR}$ & $10 \mathrm{R}$ & $\begin{array}{c}\text { Superficial Rojo oscuro y sub. } \\
\text { Superficial rojo. }\end{array}$ \\
\hline Sector pecuario1 & $2.5 \mathrm{YR}$ & $7.5 \mathrm{YR}$ & $\begin{array}{c}\text { Superficial Rojo oscuro y sub. } \\
\text { Superficial pardo. }\end{array}$ \\
\hline Sector pecuario 2 & $7.5 \mathrm{YR}$ & $5 \mathrm{YR}$ & $\begin{array}{c}\text { Superficial rojo oscuro y sub. } \\
\text { Superficial amarrillo rojizo. }\end{array}$ \\
\hline
\end{tabular}

Los suelos de la finca académica Snaky presentaron predominantemente suelos de color rojos amarrillo y pardo, esto es un indicador de suelos que presentan óxidos con diferentes grados de deshidratación. De acuerdo a los colores pardos que presentan los suelos sub superficiales del área de bosque de la finca académica Snaky, estos son indicadores de acumulaciones de óxidos de hierro parcialmente deshidratados con materia orgánica y arcillas, esta compleja asociación hace que el suelo sea muy estable, de buen drenaje y aireación.

Los suelos de la superficie de área agrícola presentan colores pardos y rojos oscuros como lo antes mencionados. Los suelos pardos son suelos que presentan óxidos de hierro parcialmente deshidratados junto con materia orgánica mientras que los suelos rojizos son suelos de presencia de óxidos de hierro parcialmente deshidratados y de dióxido de manganeso, esto es debido a un medio oxidante que permite la estabilidad de estos compuestos. Los suelos del área pecuaria (superficial) presentan colores rojizos y es un indicador de la presencia de minerales de manganeso.

\section{Características químicas de los suelos}

pH

Tabla 8: Potencial de hidrogeno $(\mathrm{pH})$.

\begin{tabular}{|l|l|l|l|l|l|l|}
\hline \multicolumn{1}{|c|}{ Bosque 1 } & \multicolumn{1}{c|}{ Bosque 2 } & \multicolumn{1}{c|}{ Bosque 3 } & \multicolumn{1}{c|}{ Agrícola 1 } & \multicolumn{1}{c|}{ Agrícola 2 } & \multicolumn{1}{c|}{ Past0 1 } & \multicolumn{1}{c|}{ Pasto 2 } \\
\hline 6.1 & 5.7 & 5.6 & 5.9 & 5.9 & 6.3 & 6.3 \\
\hline $\begin{array}{l}\text { Ligeramente } \\
\text { acido }\end{array}$ & $\begin{array}{l}\text { Medianamente } \\
\text { acido }\end{array}$ & $\begin{array}{l}\text { Medianamente } \\
\text { acido }\end{array}$ & $\begin{array}{l}\text { Medianamente } \\
\text { acido }\end{array}$ & $\begin{array}{l}\text { Medianamente } \\
\text { acido }\end{array}$ & $\begin{array}{l}\text { Ligeramente } \\
\text { acido }\end{array}$ & $\begin{array}{l}\text { Ligeramente } \\
\text { acido }\end{array}$ \\
\hline
\end{tabular}


En los suelos de la finca Snaky se observan rangos de 5.6 a 6.3, de pH, el cual es un indicador de levemente baja acides. Estos suelos por su carácter y un buen manejo con abonos órganos pueden ser apropiados para el crecimiento de los cultivos.

De acuerdo los cultivos que toleran acidez podemos encontrar la caña de azúcar con rangos 5.5 a 8.0, las musáceas 5.0 a 8.0, sandias 5.0 a 6.0, yuca, piña, pastos 4.8 a 5.8, frijol, maíz y arroz 5.6 a 6.4 .

\section{Materia orgánica en la finca Snaky}

Tabla 9: Acumulación de Materia orgánica por ecosistema.

\begin{tabular}{|l|l|l|l|l|l|l|}
\hline Bosque 1 & \multicolumn{1}{|c|}{ Bosque 2 } & \multicolumn{1}{|c|}{ Bosque 3 } & Agrícola 1 & Agrícola 2 & Pasto 1 & Past0 2 \\
\hline 0.80 & 1.14 & 0.64 & 0.15 & 1.30 & 1.84 & 0.77 \\
\hline Bajo & Bajo & bajo & bajo & bajo & bajo & bajo \\
\hline
\end{tabular}

Según Gutiérrez (2010), los rangos normales de acumulación de materia orgánica de manera general son de 1.8 es bajo, de 1.9 - 4.2 es medio y más de 4.2 es alto. En el área de bosque de la finca académica se observa una baja acumulación de materia orgánica en el suelo que anda entre el rango de 0.64 a 1.14, generando un promedio de 1.78. Esta baja cantidad de materia orgánica tiene su posible explicación por el ecosistema lluvioso de nuestra región que permite un lavado constante del mismo hacia los ríos y cauces naturales del área.

\section{Macro nutriente}

De acuerdo Torres los macro nutrientes son importantes para el suelo ya que ayudan al crecimiento de las plantas, afirman el color de hojas y desarrollan mayor resistencia a las enfermedades.

\section{Nitrógeno}

Tabla 10: Nitrógeno disponible finca académica Snaky.

\begin{tabular}{|l|l|l|l|l|l|l|}
\hline Bosque 1 & Bosque 2 & Bosque 3 & Agrícola 1 & Agrícola 2 & Pasto 1 & Pasto 2 \\
\hline $0.04 \%$ & $0.06 \%$ & $0.03 \%$ & $0.06 \%$ & $0.06 \%$ & $0.09 \%$ & $0.04 \%$ \\
\hline $\begin{array}{l}\text { Muy } \\
\text { bajo }\end{array}$ & Bajo & $\begin{array}{l}\text { Muy } \\
\text { Bajo }\end{array}$ & Bajo & Bajo & $\begin{array}{l}\text { Leve } \\
\text { bajo }\end{array}$ & $\begin{array}{l}\text { Muy } \\
\text { bajo }\end{array}$ \\
\hline
\end{tabular}

De manera general se observa una significativa deficiencia de nitrógeno en los suelos de la finca académica. Es de mencionar que los cultivos requieren de cantidades altas de nitrógeno para favorecer el crecimiento vegetativo y el color de las hojas principalmente (Castro, 2009). 
Esto tiene su explicación en que el nitrógeno es un elemento muy móvil e inestable y cuando ocurren las lluvias constantes tropicales este es arrastrado por las aguas superficiales y subterráneas.

\section{Fósforo}

Los resultados de las muestras recolectadas en la finca académica reflejan lo siguiente:

Tabla 11: Fosforo disponible finca académica Snaky.

\begin{tabular}{|c|c|c|l|l|l|l|}
\hline \multicolumn{1}{|c|}{ Bosque 1 } & \multicolumn{1}{c|}{ Bosque 2 } & \multicolumn{1}{c|}{ Bosque 3 } & \multicolumn{1}{c|}{ Agrícola 1 } & \multicolumn{1}{c|}{ Agrícola 2 } & \multicolumn{1}{c|}{ Pasto 1 } & \multicolumn{1}{c|}{ Pasto 2 } \\
\hline $8.6 \mathrm{ppm}$ & $4.1 \mathrm{ppm}$ & $5.5 \mathrm{ppm}$ & $8.2 \mathrm{ppm}$ & $6.5 \mathrm{ppm}$ & $3.5 \mathrm{ppm}$ & $4.3 \mathrm{ppm}$ \\
\hline Muy bajo & Muy Bajo & Muy Bajo & Bajo & Bajo & Muy bajo & Muy bajo \\
\hline
\end{tabular}

Según Vicent (1970), el fósforo es un macro-elemento esencial para el crecimiento de las plantas. El fósforo participa en los procesos metabólicos, tales como la fotosíntesis, la transferencia de energía y la síntesis y degradación de los carbohidratos. El fósforo se encuentra en el suelo en compuestos orgánicos y en minerales.

No obstante, la cantidad del fósforo disponible en el suelo de la finca académica Snaky es muy baja por las constante lluvias y por suelos acido de la región, por lo tanto, estos tienen que ser fertilizados en forma de abono químico u orgánico para satisfacer los requerimientos nutricionales del cultivo de interés.

\section{Potasio}

Los resultados de las muestras recolectadas en los diferentes sitios de la finca académica reflejan lo siguiente:

Tabla 12: Potasio disponible finca académica Snaky.

\begin{tabular}{|c|c|c|c|c|c|c|}
\hline Bosque 1 & Bosque 2 & Bosque 3 & Agrícola 1 & Agrícola 2 & Pasto 1 & Pasto 2 \\
\hline $\begin{array}{c}0.4 \mathrm{meq} / 100 \\
\mathrm{gr}\end{array}$ & $\begin{array}{c}0.5 \mathrm{meq} / 100 \\
\mathrm{gr}\end{array}$ & $\begin{array}{c}0.5 \mathrm{meq} / 100 \\
\mathrm{gr}\end{array}$ & $\begin{array}{c}0.7 \mathrm{meq} / 100 \\
\mathrm{gr}\end{array}$ & $\begin{array}{c}0.7 \mathrm{meq} / 100 \\
\mathrm{gr}\end{array}$ & $\begin{array}{c}0.6 \mathrm{meq} / 100 \\
\mathrm{gr}\end{array}$ & $\begin{array}{c}0.6 \mathrm{meq} / 100 \\
\mathrm{gr}\end{array}$ \\
\hline alto & Alto & Alto & Alto & Alto & Alto & Alto \\
\hline
\end{tabular}

Según Hardy et al. (1973), el potasio es uno de los tres nutrientes minerales que necesitan las plantas en mayor cantidad. Los cultivos extraen grandes cantidades de potasio del suelo para su crecimiento y desarrollo y como es de esperarse, la falta de éste elemento, influye negativamente en el rendimiento y calidad del cultivo.

A excepción de los demás nutrientes del suelo, el potasio es el único que se presenta en altos valores. Probablemente esta fue una de las razones por la cual las 
trasnacionales se establecieron con las bananeras, considerando que dicho cultivo demanda mucho del potasio.

\section{Calcio}

Los resultados de cantidad de calcio acumulado en las muestras recolectadas en la finca académica reflejan lo siguiente:

Tabla 13: Calcio disponible finca académica Snaky.

\begin{tabular}{|c|c|c|c|c|c|c|}
\hline Bosque 1 & Bosque 2 & Bosque 3 & Agrícola 1 & Agrícola 2 & Pasto 1 & Pasto 2 \\
\hline $\begin{array}{c}6.3 \mathrm{meq} / 100 \\
\mathrm{gr}\end{array}$ & $\begin{array}{c}5.4 \\
\mathrm{meq} / 100 \mathrm{gr}\end{array}$ & $\begin{array}{c}6.3 \mathrm{meq} / 100 \\
\mathrm{gr}\end{array}$ & $\begin{array}{c}9.2 \mathrm{meq} / 100 \\
\mathrm{gr}\end{array}$ & $\begin{array}{c}8.9 \mathrm{meq} / 100 \\
\mathrm{gr}\end{array}$ & $\begin{array}{c}7.3 \mathrm{meq} / 100 \\
\mathrm{gr}\end{array}$ & $\begin{array}{c}7.8 \mathrm{meq} / 100 \\
\mathrm{gr}\end{array}$ \\
\hline medio & Medio & Medio & Medio & Medio & Medio & Medio \\
\hline
\end{tabular}

Según Makeyev y Berkgaut (1989), el contenido en calcio de los diferentes tipos de suelos varía ampliamente dependiendo principalmente de los materiales de origen y del grado en que la meteorización y la lixiviación han influenciado el proceso de edificación.

En el suelo de la finca Snaky se presenta un nivel de calcio regular. Esto podría deberse a que el $\mathrm{Ca}$ (calcio) existe como un catión y gobernado por los fenómenos del intercambio catatónico. El se mantiene adherido como $\mathrm{Ca}++$ intercambiable en la superficie de los coloides cargados negativamente, por lo que se encuentra disponible en la mayoría de los suelos tropicales.

\section{Magnesio}

Tabla 14: Magnesio disponible finca académica Snaki.

\begin{tabular}{|c|c|c|c|c|c|c|}
\hline Bosque 1 & Bosque 2 & Bosque 3 & Agrícola 1 & Agrícola 2 & Pasto 1 & Pasto 2 \\
\hline $\begin{array}{c}1.7 \\
\text { meq/100 } \\
\mathrm{gr}\end{array}$ & $\begin{array}{c}1.8 \\
\mathrm{meq} / 100 \mathrm{gr}\end{array}$ & $\begin{array}{c}2.3 \\
\mathrm{meq} / 100 \\
\mathrm{gr}\end{array}$ & $\begin{array}{c}3.5 \\
\mathrm{meq} / 100 \\
\mathrm{gr}\end{array}$ & $\begin{array}{c}3.5 \\
\mathrm{meq} / 100 \\
\mathrm{gr}\end{array}$ & $\begin{array}{c}2.3 \\
\mathrm{meq} / 100 \\
\mathrm{gr}\end{array}$ & $\begin{array}{c}2.3 \\
\mathrm{meq} / \mathbf{1 0 0} \\
\mathrm{gr}\end{array}$ \\
\hline bajo & bajo & Medio & Medio & Medio & Medio & Medio \\
\hline
\end{tabular}

En los suelos de la finca Snaky se encuentran en niveles de bajo a medio. Esto podría ser debido a que son lavados rápidamente por las constantes lluvias de la región, la cual son nutrientes que se encuentra en niveles bajo en suelos ácidos. 


\section{Micronutrientes}

Los micronutrientes son tan importantes para las plantas como los nutrientes primarios y secundarios, a pesar de que la planta los requiere solamente en cantidades muy pequeñas. Sin embargo, la ausencia de cualquiera de estos micronutrientes en el suelo puede limitar el crecimiento de la planta, aún cuando todos los demás nutrientes esenciales estén presentes en cantidades adecuadas.

\section{Hierro}

Los resultados de las muestras recolectadas en la finca académica reflejan lo siguiente:

Tabla 15: Hierro disponible finca académica Snaky.

\begin{tabular}{|l|l|l|l|l|l|l|}
\hline \multicolumn{1}{|c|}{ Bosque 1 } & \multicolumn{1}{c|}{ Bosque 2 } & \multicolumn{1}{c|}{ Bosque 3 } & \multicolumn{1}{c|}{ Agrícola 1 } & \multicolumn{1}{c|}{ Agrícola 2 } & \multicolumn{1}{c|}{ Pasto 1 } & \multicolumn{1}{c|}{ Pasto 2 } \\
\hline $125.8 \mathrm{ppm}$ & $92.8 \mathrm{ppm}$ & $132.8 \mathrm{ppm}$ & $229.7 \mathrm{ppm}$ & $221.1 \mathrm{ppm}$ & $193.1 \mathrm{ppm}$ & 211.4 ppm \\
\hline Alto & Alto & Alto & Muy Bajo & Muy alto & Muy alto & Muy alto \\
\hline
\end{tabular}

El hierro en la finca académica está en el rango de alto a muy alto, y esto está íntimamente relacionada con la acides de los mismos suelo. De igual manera los colores amarillo-pardos de ciertos lugares de la finca presentan acumulaciones de óxidos hidratados como la goethita, que derivan en suelos ácidos también.

\section{Cobre}

Los resultados de las muestras recolectadas en la finca académica reflejan lo siguiente:

Tabla 16: Cobre disponible finca académica Snaky.

\begin{tabular}{|l|l|l|l|l|l|l|}
\hline \multicolumn{1}{|c|}{ Bosque 1 } & \multicolumn{1}{|c|}{ Bosque 2 } & \multicolumn{1}{c|}{ Bosque 3 } & \multicolumn{1}{c|}{ Agrícola 1 } & \multicolumn{1}{c|}{ Agrícola 2 } & \multicolumn{1}{c|}{ Pasto 1 } & \multicolumn{1}{c|}{ Pasto 2 } \\
\hline $2.7 \mathrm{ppm}$ & $1.8 \mathrm{ppm}$ & $3.5 \mathrm{ppm}$ & $4.2 \mathrm{ppm}$ & $4.2 \mathrm{ppm}$ & $4.2 \mathrm{ppm}$ & $4.3 \mathrm{ppm}$ \\
\hline bajo & bajo & Medio & Medio & Medio & Medio & Medio \\
\hline
\end{tabular}

Según Morales (2009), los suelos tropical húmedo son bajo en cobre debido a que existe una fuerte retención de la materia orgánica, en este sentido el papel de los microorganismo del suelos es básico para la descomposición de la materia orgánica, pero también la acción mayor o menor de los microorganismos depende mucho de la acides de los suelos. En el caso de la finca académica los suelos mantienen niveles de bajo a medio del elemento cobre. 


\section{Zinc}

Los resultados de las muestras recolectadas en la finca académica reflejan lo siguiente:

Tabla 17: Zinc disponible finca académica Snaky.

\begin{tabular}{|l|l|l|l|l|l|l|}
\hline \multicolumn{1}{|c|}{ Bosque 1 } & \multicolumn{1}{c|}{ Bosque 2 } & \multicolumn{1}{c|}{ Bosque 3 } & \multicolumn{1}{c|}{ Agrícola 1 } & \multicolumn{1}{c|}{ Agrícola 2 } & \multicolumn{1}{c|}{ Pasto 1 } & \multicolumn{1}{c|}{ Pasto 2 } \\
\hline 2.7 ppm & $2.4 \mathrm{ppm}$ & $3.2 \mathrm{ppm}$ & $8.5 \mathrm{ppm}$ & $8.6 \mathrm{ppm}$ & $3.8 \mathrm{ppm}$ & $4.3 \mathrm{ppm}$ \\
\hline Bajo & Bajo & Medio & Medio & Medio & Medio & Medio \\
\hline
\end{tabular}

El zinc, esencial para el crecimiento y reproducción de la planta, ayuda en el desarrollo y en la resistencia contra las enfermedades bacterianas y fungosas principalmente. En el caso de los suelos de la finca se encuentra en niveles de bajo a medio y esto generalmente repercute en los rendimientos de los cultivos. Ya que no contiene mucha proteína y hormonas de crecimiento la cual dificulta el proceso reproductivo necesario para el desarrollo del polen y el desarrollo del fruto, la cual tiende a tener hojas pequeñas e angostas.

\section{Manganeso}

Los resultados de las muestras recolectadas en la finca académica reflejan lo siguiente:

Tabla 18: Manganeso disponible finca académica Snaky.

\begin{tabular}{|l|l|l|l|l|l|l|}
\hline Bosque 1 & \multicolumn{1}{|c|}{ Bosque 2 } & \multicolumn{1}{c|}{ Bosque 3 } & \multicolumn{1}{c|}{ Agrícola 1 } & \multicolumn{1}{c|}{ Agrícola 2 } & \multicolumn{1}{c|}{ Pasto 1 } & \multicolumn{1}{c|}{ Pasto 2 } \\
\hline 66.0 ppm & $24.5 \mathrm{ppm}$ & $32.0 \mathrm{ppm}$ & $78.1 \mathrm{ppm}$ & $74.6 \mathrm{ppm}$ & $66.2 \mathrm{ppm}$ & $72 \mathrm{ppm}$ \\
\hline Muy Alto & Alto & Alto & Muy Alto & Muy alto & Muy alto & Muy alto \\
\hline
\end{tabular}

Los suelos de la finca académica mantienen niveles de alto a muy altos de manganeso. El Mn tiene funciones en el sistema enzimático de la planta, así como un rol en varias reacciones metabólicas importantes incluyendo la conversión del nitrógeno en forma de nitratos, una forma que la planta pueda utilizar.

El Mn también participa en la fotosíntesis al ayudar a la síntesis de la clorofila. Debido a esta función, los síntomas de deficiencia de Mn generalmente incluyen el amarillamente o clorosis de la hoja. Sin embargo, los altos niveles de Mn como es el caso de la finca académica y relacionada con bajos niveles de $\mathrm{pH}$, producen toxicidad en los cultivos y se refleja en la calidad y rendimiento de los de las plantas. 


\section{Conclusiones}

El estudio refleja que los suelos de la finca Snaky URACCAN, son propiedades físicas consideradas apropiadas para el desarrollo de cualquier cultivo. Su densidad aparente es baja con una textura franco a franco arenoso y los colores del suelo son de amarrillo rojizo a pardos. Sin embargo, sus propiedades químicas presentan deficiencias significativas.

En el caso de las propiedades químicas macro nutrientes, el nitrógeno y el fósforo presentaron niveles muy bajos de los recomendables para el desarrollo de cualquier cultivo, no así el potasio que presentó altos niveles. En el caso de las características químicas micronutrientes, el calcio presentó niveles regulares, mientras que el hierro, magnesio y manganeso presentaron niveles altos y que coinciden con la alta acides del suelo.

\section{Lista de referencias}

Bullock Danny (2000). Análisis de suelos. Algunas ideas acerca de presión y producción bajo siembra. Conferencias presentadas en el XVII congreso argentino de Ciencias de suelo.

Campillo, R. (1994). Diagnóstico de la fertilidad de los suelos de la Décima Región. En: Campillo y Bortolameolli (ed). Corrección de la fertilidad y uso de enmiendas en praderas y cultivos forrajeros. Serie Remehue $\mathrm{N}^{\circ} 53$. Instituto de Investigaciones Agropecuarias, Centro Regional de Investigación Remehue, Osorno, Chile. 135 p.

Charlton, L.; Hampton, G. y Scott, .J. (1986). Efectos de fertilización sobre suelos ácidos. Actas de la Nueva Zelanda. Asociación de Agrónomos, 47: 165-172.

Cisternas, E. (1992). Biología y control de insectos plagas en praderas. En: Seminario Manejo de praderas permanentes, Estación Experimental Remehue, Osorno, Chile, -117 .

Cuevas, E. y Balocchi, O. (1983). Producción de forraje. Serie B-7. Instituto de Producción Animal, Universidad Austral de Chile, Valdivia. 201 pp.

De la Rosa, Diego. (2008). Evaluación Agro-Ecológica de Suelos, para un Desarrollo Rural Sostenible. Ediciones Mundi-Prensa. $1^{\mathrm{a}}$. Edición. Madrid.

Dexter, et al (2004). La predicción de las estructuras del suelo producidos por la labranza.

Escobar, D. y Rodríguez de Vega A. (2009). Tabla de interpretación de análisis de suelos. Centro de Investigaciones Agronómicas, Universidad de Costa Rica. Mimeo. 
Etchevers B., J. P. Anzastiga, V. Volke y G. Etchevers. (1986). Correlación y calibración de métodos químicos para la determinación de fósforo disponible en suelos del Estado de Puebla. Agrociencia 65: 161- 178.

Etchevers B. J. (1991). La función del laboratorio en el diagnóstico de la necesidad de fertilizantes. Enfoques tradicionales y modernos del análisis químico de suelos. ADIFAL marzo - abril: $21-27$.

Fasbender, H. (1985). Quimica de suelos: con énfasis en América Latina. 123 p.

Foth, D; Henry. (1985). Fundamentos de la Ciencia del Suelo. Editorial CONTINENTAL, S.A, MX. 265 p.

Guerrero, G., Andrés (1996). El suelo, los abonos y la fertilización de los cultivos. Ediciones Mundi - Prensa. Madrid. 